\title{
Empirical Validation of MoDe4SLA; Approach for Managing Service Compositions
}

\author{
Lianne Bodenstaff ${ }^{\star 1}$, Andreas Wombacher ${ }^{1}$, and Manfred Reichert ${ }^{2}$ \\ 1 University of Twente, The Netherlands \\ $\{1$.bodenstaff, a.wombacher\} @utwente.nl \\ 2 University of Ulm, Germany \\ manfred.reicherteuni-ulm.de
}

\begin{abstract}
For companies managing complex Web service compositions, challenges arise which go far beyond simple bilateral contract monitoring. For example, it is not only important to determine whether or not a component (i.e., Web service) in a composition is performing properly, but also to understand what the impact of its performance is on the overall service composition. To tackle this challenge, in previous work we developed MoDe4SLA which allows managing and monitoring dependencies between services in a composition. This paper empirically validates MoDe4SLA through an extensive and interactive experiment among 34 participants.
\end{abstract}

Key words: SLAs, service composition, empirical validation, SLA management

\section{Introduction}

Regarding the monitoring of Web service (WS) compositions, it is necessary to take composition structure as well as characteristics of services into account. This information is needed to assess composition performance. This is particularly important when considering the growing complexity of WS compositions. Particularly, providers of composite services struggle to manage these complex constellations. Different services are provided with different quality levels. Further, services stem from different providers, and have different impact on the composition. To meet Service Level Agreements (SLA) with its customers any company faces the challenge of managing its underlying services. For each SLA violation the company determines its impact on the composition and decides on how to respond. Generally, complexity of this decision process grows with the number of services being involved in the composition.

The goal of MoDe4SLA [1, 2] is to determine for each service in a composition its impact on the composition performance. The latter is measured by analyzing different metrics (e.g. costs and response time) as used in SLAs. Through analysis of both dependency structure and impact it becomes possible to monitor composition performance taking dependencies between services into account. The advantage of such analysis is the possibility to explain SLA violations of a service composition through identifying badly performing services the composition depends on.

* This research has been supported by the Dutch Organization for Scientific Research (NWO) under contract number 612.063.409 
This paper validates our scientific solution (MoDe4SLA) for the real-life problem of managing complex service compositions through a controlled, quantitative experiment $[3,4]$. Experimental validation in Computer Science is recognized as being very important $[5,4]$. However, still a minority of research papers actually provides some experimental results $[6,7]$. Without validating developed approaches like MoDe4SLA, however, a researcher might steer his research efforts into a fruitless direction [5]. In our evaluation we conduct an experiment with 34 participants. These participants are asked to manage Web service compositions using our approach and to do this without using MoDe4SLA. We gather data on their experiences and analyze them.

This paper evaluates usefulness of our MoDe4SLA approach for managers burdened with maintenance of service compositions [8]. We first provide some background information on MoDe4SLA in Section 2. Related work is discussed in Section 3. We evaluate usefulness by asking experts to manage simulated runs of service compositions using MoDe4SLA (Sections 4 and 5). We conclude with a summary in Section 6.

\section{Background \& Example scenario}

MoDe4SLA [1, 2] intends to supports companies in managing their composite services by identifying and monitoring their dependency on other services requested from external providers. Our approach pinpoints to services causing SLA violations of the composition. SLAs describe constraints (e.g., response time and availability) a service has. Our abstract example scenario (Fig. 1) depicts a composition where every composition invocation triggers WS 1 - WS 7, WS 13, WS 16, and WS 17. In addition WS 8, WS 12, or the Loop-construct is chosen (XOR-construct where each outgoing edge is annotated with the chance to be chosen compared to its siblings). If the Loop is chosen, WS 9 WS 11 are invoked in a repeated sequence (on average 3 times). Further, either WS 14 or WS 15 is invoked (ORDISC-construct with ratio $0.89: 0.11)$. Except for services in the Loop sequence, all services are invoked in parallel.

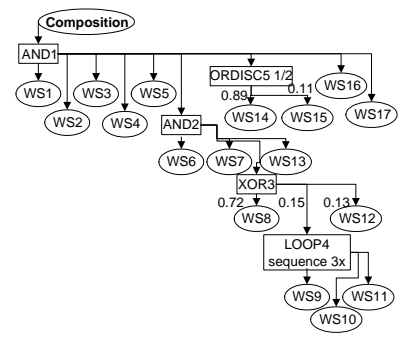

Fig. 1. Exemplary service composition



Fig. 2. Estimated impact tree for cost

Offering a composite service to customers implies that a company relies on other service providers. SLA constraints might depend on different services, or depend on them in different ways. For example, if a company offers information with fast response time by querying five providers and returning information of the fastest responding one, 
a cost constraint is influenced by all five services (invoking means paying), while a response time constraint is only influenced by the fastest responding one. As a consequence, MoDe4SLA analyzes dependencies for each constraint separately and represents them in impact trees. We analyze expected behavior of services using their SLAs and realized behavior using logs. Differences between realized and expected behavior are presented in a feedback tree to users. MoDe4SLA calculates three values for these trees: contribution factors depict the number of times a branch is invoked and contributes to the constraint value. Service contributions depict the average constraint value when it contributes, and impact factors combine these measures.

Contribution factors. Each branch of a tree is annotated with a value indicating the number of times the branch contributes to the composition per composition invocation. Fig. 2 depicts the estimated impact tree for costs based on the composition structure from Fig. 1 and estimations on the number of invocations. For example, each outgoing edge of the XOR-construct has an expected contribution factor. E.g., the composition manager expects WS 8 to contribute $72 \%$ of the time to the composition costs.

Service contribution. For each service we calculate its expected contribution to overall response time or cost of the composition by using its SLA constraints. For the realized average contribution, we use these invocations of the service that contributed to overall costs or re-

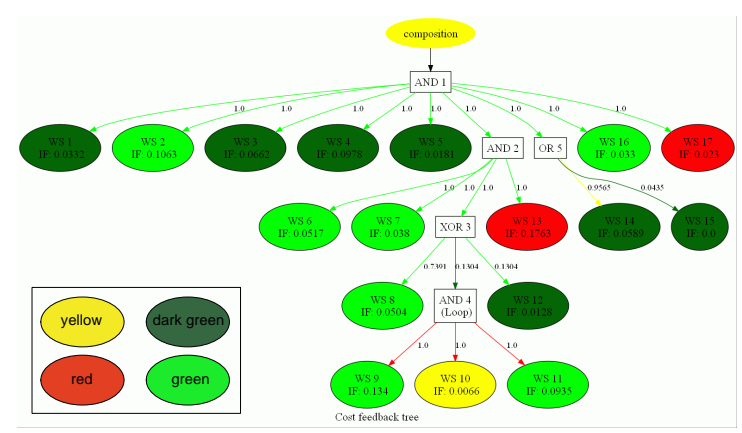

Fig. 3. Cost feedback tree sponse time. Consequently, not every invoked service is considered and, therefore, our approach does not substitute bilateral monitoring.

Impact factors. Each service is annotated with an impact factor (IF in Fig. 2). This is calculated by multiplying contribution factor (i.e., number of times a service contributes per composition invocation) and average service contribution (i.e., average constraint value of a service, e.g., average response time), divided by the average constraint value (e.g., response time) of the composition. The value indicates the average contribution percentage to the composition concerning a constraint. For example, in Fig. 2, WS 13 has an expected IF of 0.1675 , i.e., it is expected that $16.75 \%$ of the composition costs are due to WS 13. Generally, impact factors in one composition add up to 1.

Feedback trees. Our goal is to provide information on composition performance through graphical feedback trees. These trees (cf. Fig. 3) indicate differences between design time estimations and realized values monitored in event logs. More particular, the feedback tree shows causes for SLA violations of a composite service. These differences are depicted by coloring branches for contribution factors and services for service 
contributions. As discussed, impact factors combine these two measures and show the average impact of each service. Color red indicates worse performance than expected and green indicates proper performance. Yellow indicates the service is not performing perfectly, but still within boundaries set by the company, and dark green indicates a service runs better than anticipated on. Edges are colored in the same manner. For example, red indicates an edge contributes more often than expected.

Fig. 3 depicts the cost feedback tree of our example scenario. The yellow composition indicates the cost constraint is not met. This is caused by two factors: (1) Performance of service WS 13 is bad since it violates its SLA (i.e., it is colored red) with an impact factor (IF) of 0.1763 , i.e., almost $18 \%$ of the overall costs are contributed by this service. (2) Due to the structure of the composition, services WS 9 - WS 11 are contributing more often than expected (i.e., red incoming edges) which causes elevated costs. Although WS 17 is not functioning properly, its impact factor is too low (i.e., $2 \%$ ) for causing major composition violations. Furthermore, several services are overperforming (i.e., dark green). If performance problems are solved, these services might positively influence overall costs.

\section{Related work}

Menasce [9] presents response time analysis of composed services to identify impact of slowed down services. The result is a measure for the overall slow down depending on statistical likelihood of a service not delivering expected response time. As opposed to our approach, Menasce performs analysis at design-time rather than providing a runtime based analysis. A different approach with the same goal is the virtual resource manager proposed by Burchard et al. [10]. It targets a grid environment where a calculation task is distributed among different grid vertices for individual computation jobs. If a grid vertex fails to deliver the promised service level, a domain controller first reschedules the job onto a different vertex within the same domain. If this action fails, the domain controller attempts to query other domain controllers for passing over the computation job. Although the approach covers runtime, it follows a hierarchical autonomic recovery mechanism. MoDe4SLA focusses on identifying causes for correction on the level of business operations rather than on autonomous job scheduling. In the COSMA approach Ludwig et al. [11] describe a framework for life cycle management of SLAs in composite services. They recognize the problem of managing dependencies between different SLAs. Furthermore, their COSMAdoc component describes composite specific dependencies but does not explicate what type of dependencies are considered. The SALMon approach by Oriol et al. [12] aims at monitoring and adapting SOA systems at runtime. Monitoring is done for SLA violations. Further, a decision component performs corrective actions so that SLAs are satisfied. Their approach does not focus on service compositions but on runtime adaptability. As a consequence, they are not concerned with dependencies between different SLAs. Moser et al. [13] describe an approach for automatically replacing services at runtime without causing any downtime for the overall system. The BPEL processes are monitored according to their QoS attributes and replacement of services and partners is offered on various strategies. Although their approach has similarities to ours, their goals focus on runtime adaptability, 
and not on service compositions and their SLA dependencies. Sahai et al. [14] aim at automated SLA monitoring by specifying SLAs and not only considering provider side guarantees but focus also on distributed monitoring, taking the client side into account as well. Barbon et al. [15] enable run-time monitoring while separating business logic from monitoring functionality. For each process instance a monitor is created. Unique for this approach is its ability to also monitor classes of instances, enabling abstraction from an instance level. The smart monitoring approach of Baresi et al. [16] implements the monitor itself as a service. There are three types of monitors available for different aspects of the system. Their approach is developed to monitor specifically contracts with constraints. In [17] Baresi et al. present an approach to dynamically monitor BPEL processes by adding monitoring rules to the different processes. These rules are executed during runtime. Our approach does not require modifications to the process descriptions what might suit better to some application areas. An interesting approach in this direction is work by Mahbub et al. [18] who considers the whole state of the system in their monitoring approach. They aim at monitoring derivations of system behavior.

Most of the discussed approaches are evaluated by providing a proof-of-concept implementation (e.g., $[19,20])$. In addition, some approaches are validated by a performance study (e.g., $[18,21])$. However, to our knowledge, none of the approaches has been empirically validated. This complicates finding a suitable method to compare our approach with. Therefore, we choose to use straightforward bilateral monitoring as a baseline for evaluating MoDe4SLA.

\section{Evaluating usefulness: Setup}

The design of our evaluation is described more extensively in Bodenstaff et al. [8]. To evaluate usefulness of the results from our MoDe4SLA analysis, we interview experts, asking them to make a statement on how useful they perceive the approach when managing compositions. We use the following criterion to evaluate usefulness:

MoDe4SLA is considered as being useful when experts testing it perceive the feedback given by MoDe4SLA as more useful for managing and maintaining the composition than when using bilateral monitoring results.

Common management approaches return bilateral monitoring results to users. They do not provide information on the relation between the different services, but merely return individual service performance. For evaluating our MoDe4SLA approach, we extend the implementation of an existing simulator - SENECA [22] - with generating impact models and with an analyzer module (cf. Fig. 4). This simulator randomly generates a composition structure for a given number of services. To each created service in the composition a randomly generated SLA is assigned. The impact models are derived based on the composition structure and SLAs. SENECA simulates invocation of the services according to the composition structure. Accordingly, services might violate their SLAs. The simulator gathers runtime data and generates feedback models. 
For our evaluation we prepare three compositions of different complexity. The complete set of documents, including the questionnaire handed out to experts for evaluation can be found in Bo-

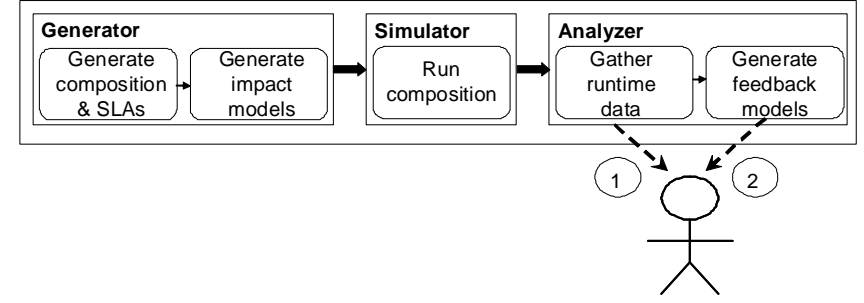

Fig. 4. Evaluating usefulness denstaff et al. [23]. The first test case (TC1) consists of five services with three constructs, the second test case consists of ten services with one OR-split and one discriminative join. Finally, the third test case consists of seventeen services connected through five constructs. This case constitutes our example scenario (cf. Fig. 1).

For each composition two documents are prepared. The MoDe4SLA document contains feedback models for both response time and costs, while the control document contains performance data for each service resulting from bilateral monitoring, but does not provide information on how they are related [23]. Main goal of our evaluation is to test following hypothesis:

The MoDe4SLA document has a clear benefit over the control document when managing the composition.

We evaluate this hypothesis by conducting a survey considering the following research questions (RQ):

RQ1 Accuracy of identifying malfunctioning services using MoDe4SLA in comparison to the use of bilateral monitoring results,

RQ2 Efficiency in identifying malfunctioning services using MoDe4SLA compared to bilateral monitoring results,

RQ3 Confidence experts have in their answers when using MoDe4SLA compared to bilateral monitoring results.

RQ4 How complex is the MoDe4SLA approach for users?

RQ5 Which possible improvements do experts suggest for the MoDe4SLA approach?

For this purpose, we prepare a questionnaire of 49 questions that experts answer before, during and after the experiment. Typically they five-level Likert item (i.e., Strongly disagree, Disagree, Neither agree nor disagree, Agree, Strongly agree) to rate responses. We start with a trial run on three colleagues to discover problems in examples, test cases, and questionnaire. Although no errors are found, a front sheet depicting graphically each composition is added. We conduct six more sessions with 34 participants from several universities and companies. Each session consists of:

1. A presentation explaining the goal of the approach.

2. Discussion of two examples where bilateral monitoring results and MoDe4SLA feedback model results are explained, including an interpretation discussion.

3. The evaluation: First some introductional questions are answered (Q1-Q7) after which the first test case with bilateral monitoring results is studied and the participant answers Q8-Q11. Then the MoDe4SLA feedback trees are studied and 
Q12-Q18 are answered. These steps are repeated for the second and third case. We conclude with general questions (Q41-Q47).

\section{Conclusions from evaluating usefulness}

We introduce some demographics after which we discuss answers related to questions from Section 4. We conclude with an analysis of relations between different outliers in questions in Section 5.3. Statistics on all questions can be found in [23].

\subsection{Demographics}

The group of 34 participants consists of experts in developing and managing services. 11 experts are from industry, of which 9 are also active in academia, and additional 23 experts from academia. $15 \%$ of the experts have experience using tools for managing composite services. $15 \%$ of the experts have experience developing tools for managing composite services. $60 \%$ of the participants have not worked with composite services, while the remaining $40 \%$ have experience varying from less than one year to over three years. $9 \%$ of the participants consider themselves having a high level of expertise in managing composite services. They are in particular supportive for MoDe4SLA approach and therefore no explicit discussion of this participant group is done. The low number of experts in managing service compositions does not influence the overall result since also experts in services understand the complexity of service compositions and their management although they have not performed the task themselves yet.

Although our participants are familiar with service compositions, on average their expertise in managing them is not high. As advantage this inexperience helps us in determining how difficult it is to master MoDe4SLA. As disadvantage, we cannot expect much feedback on possible other approaches for managing service compositions.

\subsection{Statistics}

We start each test case with a question on how complex the participants feel the composition is (TC1 with 5 services: Q8, TC2 with 10 services: Q19, TC3 with 17 services: Q30) (cf. Fig. 5). We assume TC2 is perceived as less complex than TC1 since it contains only one construct: an OR-split with discriminative join while TC1 contains three constructs. We add this question since we assume the more complex the composition is, the more useful MoDe4SLA will be. Although participants consider the different test cases to be of different complexity, and although participants appreciate using MoDe4SLA even more when considering the complex test case (i.e., TC3), these differences were lower than expected as discussed in the following.

RQ1: Accuracy. We want to know whether participants feel that identifying problematic services can be done more accurately with than without MoDe4SLA. We have two questions giving us an insight on this.

The first question is asked for each test case, and for both response time and costs. We ask participants whether they perceive identifying the impact each service has on the 




Fig. 5. The composition is complex.

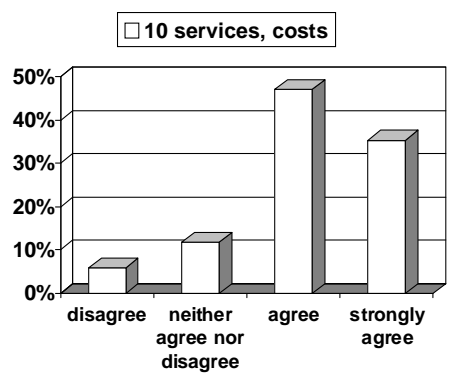

Fig. 6. It is easier to determine the impact of each service with the analysis than without.

composition, is easier with MoDe4SLA than it is without MoDe4SLA (for TC1: Q15 and Q16, for TC2: Q26 and Q27, for TC3: Q37 and Q38). MoDe4SLA is perceived as being more useful for response time than for costs, and as more useful for TC3 than for TC1 and TC2. The majority perceives the use of MoDe4SLA as very helpful for easier identification of the impact. Fig. 6 depicts the histogram with least positive responses for our approach. It still entails over $80 \%$ of the participants agreeing or strongly agreeing to the statement.

Second, for each test case we ask participants whether they consider MoDe4SLA being helpful when managing the composition with regard to accurately depicting malfunctioning services (for TC1, TC2, and TC3, and Q18, Q29, and Q40). Fig. 8 depicts results. $75-80 \%$ of the participants agree or strongly agree that MoDe4SLA is helpful to accurately depict these services even for the least complex composition.

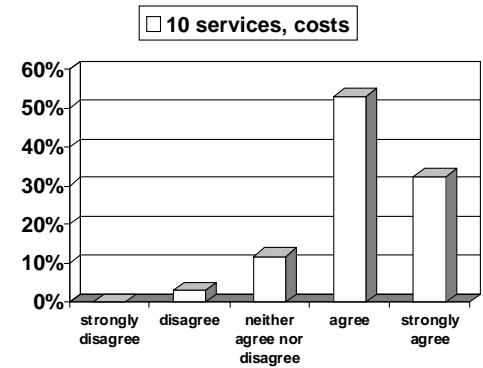

Fig. 7. It takes less time to see relations between Fig. 8. MoDe4SLA is helpful when depicting different services and the composition.

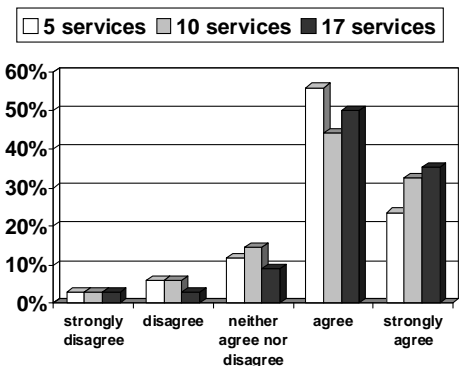

malfunctioning services.

RQ2: Efficiency. We investigate whether participants consider it as more efficient when using MoDe4SLA for managing service compositions than without. First, for each test case and for both response time and cost we ask participants to respond to the statement that it takes less time to see relations between the different services in a composition when using MoDe4SLA. Since MoDe4SLA relies on identifying relations and dependencies between the services, we assume that MoDe4SLA is helpful when try- 
ing to identify these relations. Depending on the test case, $85-100 \%$ of the participants (strongly) agree with this statement. Fig. 7 depicts the least positive responses.

Second, for each test case we ask participants whether they consider MoDe4SLA being helpful when managing the composition with regard to efficiently depicting malfunctioning services (for TC1, TC2, and TC3, and Q18, Q29, and Q40). Fig. 9 depicts results for these questions. Around $90 \%$ of the participants agree or strongly agree that MoDe4SLA is helpful to accurately identify these services.

RQ3: Confidence. To evaluate how confident participants are when making a choice on which services to adapt to get better performance, for each test case we ask three questions. First, we ask how confident they are making a choice before seeing MoDe4SLA models. Second, we ask how confident they are about their original choice when seeing the models. Third, we ask how confident they are making a choice when considering MoDe4SLA models. The aim of the second question is to find out whether participants feel MoDe4SLA giving additional support. If they feel more or less confident, MoDe4SLA apparently gives them additional insights. If they do not change their opinion, MoDe4SLA has not given additional insights. The change in confidence (i.e., the second question) is depicted in Fig. 10. For each test case at least $80 \%$ of the participants change their confidence level. The confidence level of participants before considering MoDe4SLA is depicted in Fig. 11. Experts have reasonable confidence levels in the first and second test case but no confidence in the third one. In Fig. 12 the confidence level goes up for all test cases after participants studied the MoDe4SLA files. On average participants feel more confident making choices on which services to adapt using MoDe4SLA than without it.

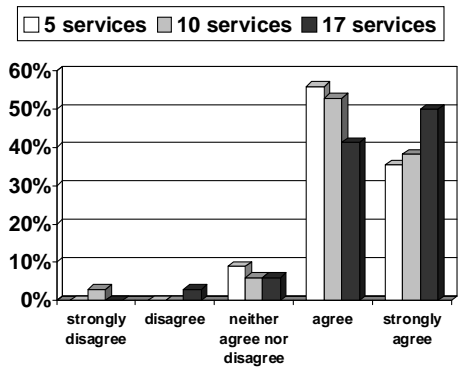

Fig. 9. MoDe4SLA is helpful to fast select ser- Fig. 10. How is your confidence in your selecvices to renegotiate.

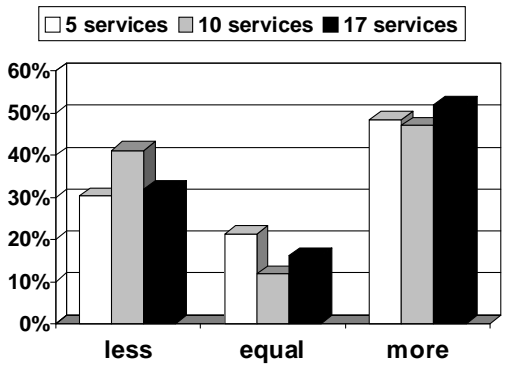

tion for renegotiation?

We test usefulness of our approach by answering RQ1-RQ3. In addition, we ask participants at the end of the survey to respond to the statement that using MoDe4SLA is helpful when managing composite services (cf. Fig. 13). None of them disagrees or strongly disagrees with this statement. $94 \%$ of them agree or strongly agree with it.

RQ4: Complexity. Another important aspect is how difficult MoDe4SLA is to comprehend. We strive to develop an intuitive approach that is easy to understand for users. Of course, the positive evaluation results concerning MoDe4SLA usefulness after a short 


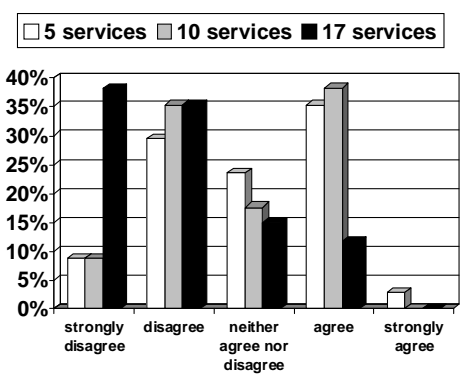

Fig. 11. I would feel confident in selecting ser- Fig. 12. I feel more confident selecting renegotivices for renegotiation.

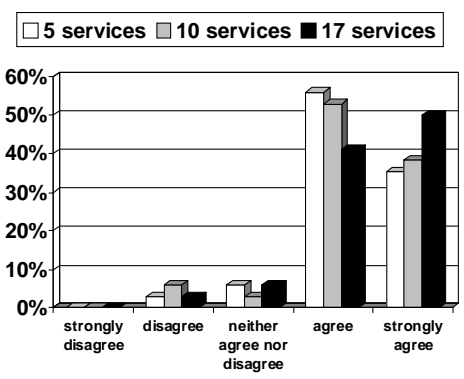
ation services with MoDe4SLA than without.

training period support our claim that its usability is good. However, we also want to know whether participants feel the given explanation of MoDe4SLA was sufficient to use the models (cf. Q42). The presentation takes at most one and a half hour, including discussion and questions. Fig. 14 indicates that over $85 \%$ of the participants agree with this statement. A considerable group (around 12\%) appreciate more explanation.

Furthermore, in Q45 we ask participants to name weak points of the approach. Here, we find indicators MoDe4SLA is less intuitive for some of the participants. 7 of them (i.e., around 20\%) indicate they have problems understanding the values in the model. With these values, participants sometimes mean impact factors, but usually contribution factors (i.e., branch annotations) turn out to be hard to understand. The magnitude of numbers confused some of the participants. 2 of them (i.e., around 6\%) state for them the feedback models are too complex to comprehend. In conclusion, about $75 \%$ of the participants have no difficulties understanding values in the feedback models.

RQ5: Possible improvements. We ask participants to state what they feel is most beneficial about the feedback models. Participants like the visualization part, especially the coloring. Furthermore, impact factors and analysis itself are beneficial. Also, we ask for possible improvements. The first is a reduction of the many numbers used in the models. As discussed in RQ4, for some participants there is too much information given. In addition, participants feel they are able to choose using colors and impact factors. Therefore, we consider filtering this information when models are presented to users. Second, participants appreciate some interpretation guidelines. For example, "a low impact factor indicates", "a high ratio means", and "from the combination of an impact factor and ratio you can derive". Therefore, we consider extending the presentation with information on these statements. Third, related to the previous improvement, participants appreciate guidelines for decision making. It is beneficial if the models indicate which services to consider for change, and why. Currently, models only provide monitoring information without suggestions on how to improve performance. Developing such guidelines is part of our future work.

\subsection{Evaluation conclusions}

Test cases. Although we introduce three test cases with different complexity levels, MoDe4SLA is already perceived as useful when managing a composition with only 


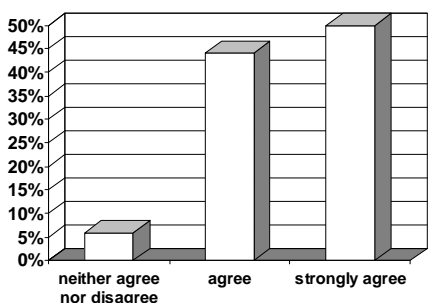

Fig. 13. MoDe4SLA is helpful for managing ser- Fig. 14. The presentation is sufficient to undervice compositions.

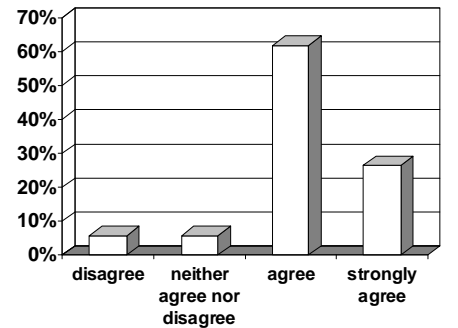

stand MoDe4SLA.

five services. Furthermore, our test case with seventeen services is considered as highly complex. However, real-life compositions are typically much larger. As a consequence, a proper management approach is definitely necessary in those cases. Further, TC2 is perceived as less complex than TC1 although it consists of twice as many services. The three constructs in TC1 make it more complex than TC2 that contains one construct.

Cost versus response time. When browsing through the different diagrams, it is clear that most participants struggle more with response time dependencies than with cost dependencies. As a result, MoDe4SLA is especially appreciated in response time models, which is also supported by answers of Q48 where beneficial parts are named. Response time of a branch depends on the interaction between different services: Whether service A contributes, does not only depend on whether it is invoked, and how fast is runs, but also on how fast its neighbor runs. Also for cost the contribution depends on the cost of other services, but this dependency is less strong.

\section{Summary and Outlook}

This paper presents our evaluation of usefulness of our MoDe4SLA approach. In several interactive sessions we conduct an extensive evaluation where 34 participants answer 49 questions. To support our hypothesis that MoDe4SLA models have a clear benefit over an approach that only supports bilateral monitoring (cf. Section 4), we investigate usefulness of our approach as perceived by experts. All three sub-questions concerning accuracy (RQ1), efficiency (RQ2), and confidence (RQ3) clearly indicate that participants benefit from the MoDe4SLA models. Though there are improvements to be considered, as discussed in RQ5, participants are able to properly understand MoDe4SLA within one and a half hour. These results give us support to continue developing our monitoring approach. So far, we only ask participants for their opinion. There are no good or bad answers. Of course, we want to know whether the answers our participants give are effective as well. In other words, we want to know whether their decisions are better when making them with MoDe4SLA than without. This is considered in our effectiveness evaluation in future research. 


\section{References}

1. Bodenstaff, L., Wombacher, A., Reichert, M., Jaeger, M.C.: Monitoring dependencies for SLAs: The MoDe4SLA approach. In: Proc. SCC'08. 21-29

2. Bodenstaff, L., Wombacher, A., Reichert, M.U., Jaeger, M.C.: Analyzing impact factors on composite services. In: Proc. SCC'09. 218-226

3. Kitchenham, B.: Evaluating Software Engineering Methods and Tool Part 1: The Evaluation Context and Evaluation Methods. ACM SIGSOFT Software Engineering Notes 21(1) (1996)

4. Zelkowitz, M., Wallace, D.: Experimental Models for Validating Computer Technology. IEEE Computer 31(5) (1998) 23-31

5. Tichy, W.: Should Computer Scientists Experiment More? IEEE Computer 31(5) (1998)

6. Sjøberg, D., Hannay, J., Hansen, O., Kampenes, V., Karahasanovic, A., Liborg, N., Rekdal, A.: A Survey of Controlled Experiments in Software Engineering. IEEE Transactions on Software Engineering 31(9) (2005) 733-753

7. Tichy, W., Lukowicz, P., Prechelt, L., Heinz, E.: Experimental Evaluation in Computer Science: A Quantitative Study. Journal of Systems \& Software 28(1) (1995) 9-18

8. Bodenstaff, L., Wombacher, A., Jaeger, M.C., Reichert, M., Wieringa, R.: Monitoring service compositions in MoDe4SLA: Design of validation. In: Proc. ICEIS'09

9. Menascé, D.A.: Response-time analysis of composite Web services. IEEE Internet Computing 8(1) (2004) 90-92

10. Burchard, L.O., Hovestadt, M., Kao, O., Keller, A., Linnert, B.: The Virtual Resource Manager: An architecture for SLA-aware resource management. In: Proc. on Cluster Comp. and the Grid. (2004) 126-133

11. Ludwig, A., Franczyk, B.: Managing dynamics of composite Service Level Agreements with COSMA. In: Proc. Int. Conf. on Fuzzy Systems and Knowledge Discovery. (2008)

12. Oriol, M., Marco, J., Franch, X., Ameller, D.: Monitoring adaptable SOA-systems using SALMon. In: Proc. WS on Service Monitoring, Adaptation and Beyond. (2008)

13. Moser, O., Rosenberg, F., Dustdar, S.: Non-intrusive monitoring and service adaptation for WS-BPEL. In: Proc. WWW'08

14. Sahai, A., Machiraju, V., Sayal, M., van Moorsel, A.P.A., Casati, F.: Automated SLA monitoring for Web services. In: Proc. Int. WS on Distributed Systems. (2002) 28-41

15. Barbon, F., Traverso, P., Pistore, M., Trainotti, M.: Run-time monitoring of instances and classes of Web service compositions. In: Proc. ICWS'06. 63-71

16. Baresi, L., Ghezzi, C., Guinea, S.: Smart monitors for composed services. In: Proc. ICSOC'04. 193-202

17. Baresi, L., Guinea, S.: Towards dynamic monitoring of WS-BPEL processes. In: Proc. ICSOC'05. 269-282

18. Mahbub, K., Spanoudakis, G.: Run-time monitoring of requirements for systems composed of Web-services: Initial implementation and evaluation experience. In: Proc. ICWS'05

19. Baresi, L., Guinea, S.: Towards dynamic monitoring of WS-BPEL processes. Lecture Notes in Computer Science 3826 (2005) 269

20. Ludwig, A., Franczyk, B.: COSMA-An Approach for Managing SLAs in Composite Services. In: Proc. ICSOC'08, Springer 632

21. Pistore, M., Traverso, P.: Assumption-Based Composition and Monitoring of Web Services. Test and Analysis of Web Services (2007) 307-335

22. Jaeger, M.C., Rojec-Goldmann, G.: SENECA - simulation of algorithms for the selection of Web services for compositions. In: Proc. TES'05. 84-97

23. Bodenstaff, L., Wombacher, A., Reichert, M.: Evaluation data for MoDe4SLA. Technical report: TR-CTIT-10-05, University of Twente (2010) 